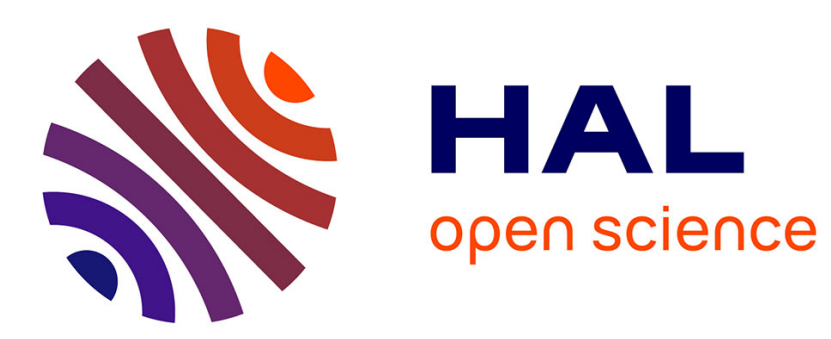

\title{
Etude d'un capteur électromagnétique plat de proximité
}

\author{
A. Billat, A. Leclerc, J.C. Emond, G. Villermain-Lecolier
}

\section{To cite this version:}

A. Billat, A. Leclerc, J.C. Emond, G. Villermain-Lecolier. Etude d'un capteur électromagnétique plat de proximité. Revue de Physique Appliquée, 1986, 21 (7), pp.443-450. 10.1051/rphysap:01986002107044300 . jpa-00245462

\section{HAL Id: jpa-00245462 https://hal.science/jpa-00245462}

Submitted on 1 Jan 1986

HAL is a multi-disciplinary open access archive for the deposit and dissemination of scientific research documents, whether they are published or not. The documents may come from teaching and research institutions in France or abroad, or from public or private research centers.
L'archive ouverte pluridisciplinaire HAL, est destinée au dépôt et à la diffusion de documents scientifiques de niveau recherche, publiés ou non, émanant des établissements d'enseignement et de recherche français ou étrangers, des laboratoires publics ou privés. 
Classification

Physics Abstracts

$06.30 \mathrm{C}-07.55$

\title{
Etude d'un capteur électromagnétique plat de proximité
}

\author{
A. Billat, A. Leclerc, J. C. Emond et G. Villermain-Lecolier \\ Laboratoire d'Applications de la Microélectronique, Université de Reims, B.P. 347, 51062 Reims Cedex, France
}

(Reçu le 24 décembre 1985, révisé le 25 mars 1986, accepté le 8 avril 1986)

\begin{abstract}
Résumé. - La partie sensible des capteurs de proximité magnétiques est souvent constituée d'une bobine enroulée autour d'un noyau. Nous avons réalisé une bobine dont la géométrie est différente. Elle permet par son incorporation dans un oscillateur, la réalisation d'un capteur de proximité peu coûteux. Nous présentons ici les caractéristiques physiques et électriques de plusieurs échantillons. Cette étude constitue une première approche d'optimisation du capteur et de son électronique associée. Nous montrons que l'on peut détecter correctement un bord de tôle ainsi que la présence de trous dans une tôle.
\end{abstract}

\begin{abstract}
The sensible part of magnetic proximity sensors is often constituted of a coil wound round a core. We have realized a flat coil of which geometry is different. On account of its incorporation into an oscillator, it allows an inexpensive manufacturing of proximity sensors. We present here the physical and electrical characteristics of different samples. This work is a first study of the sensor optimization and of its associated electronic. We demonstrate that we can accurately detect a sheet metal edge and also the presence of hole in a sheet.
\end{abstract}

\section{Introduction.}

La plupart des capteurs de proximité magnétiques actuellement commercialisés comportent une bobine enroulée autour d'un noyau. On sait alors [1] que la proximité d'une pièce métallique peut être détectée selon deux principes physiques différents.

La bobine produit dans l'espace un champ magnétique variable. Si une cible métallique non ferromagnétique est placée dans cet environnement, elle devient le siège de courants de Foucault localisés dans une couche superficielle dont l'épaisseur est d'environ trois fois celle de l'effet de peau. Ces courants réagissent sur l'impédance de l'enroulement si la modulation en fréquence est assez élevée. La proximité d'une surface métallique peut alors être déduite d'une mesure d'impédance. Si la cible est ferromagnétique, au phénomène précédemment cité, vient s'ajouter la variation de reluctance du circuit magnétique cible-capteur lors du déplacement relatif de ses éléments.

Malgré des caractéristiques de sorties fortement non linéaires ces capteurs sont très utilisés [2]. Leurs conditions de fonctionnement optimum ont été d'ailleurs abondamment décrites [3-5].

Le travail que nous présentons ici montre la possibilité d'utiliser comme élément sensible une bobine plate gravée sur circuit imprimé. Cette réalisation a l'avantage d'être simple et peu coûteuse. Un circuit oscillant utilisé comme conditionneur permet d'obtenir une relation fréquence-distance.

Le signal de sortie du capteur est alors analysé par un système à microprocesseur.

\section{Bobines plates.}

\subsection{TeChNiques De RÉalisation DeS bOBINES Plates.}

2.1.1 Réalisation physique. - Les bobines sont gravées sur circuit imprimé de $35 \mu \mathrm{m}$ d'épaisseur à partir d'un masque. La plupart des échantillons ont été gravés sur support rigide en résine époxy ou sur support souple en capton. Le masque a la forme d'une spirale (Fig. 1). Il est réalisé à partir d'un traceur de courbes Benson 1202 piloté par un ordinateur IBM série 1. Il est impératif de tracer ce masque sur un support de grande stabilité (polyester Benson) puisqu'il sera réduit d'un facteur dix environ pour donner le masque définitif. Les différentes étapes de ce travail doivent être particulièrement soignées et réalisées en atmosphère exempte de poussières.

2.1.2 Caractérisation géométrique. - La bonne qualité physique des bobines plates est vérifiée à l'aide d'une lunette binoculaire. L'épaisseur des traits et intertraits (Fig. 2) est mesurée au microscope. Une largeur de trait ou d'intertrait de l'ordre de $70 \mu \mathrm{m}$ apparait comme une limite raisonnable pour la 


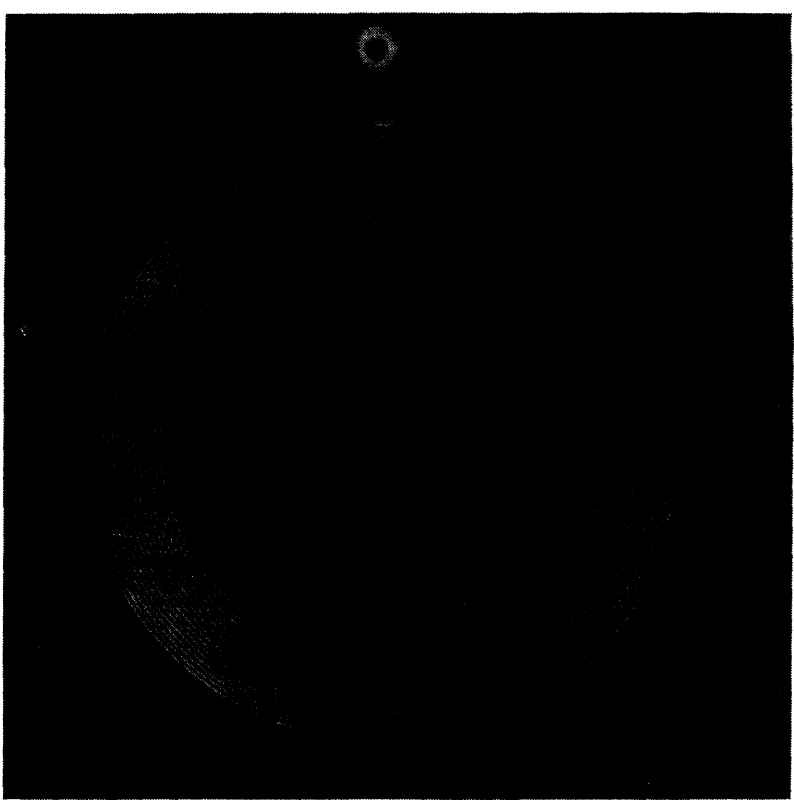

Fig. 1. - Bobine plate réalisée sur circuit imprimé.

[Flat coil realized in printed circuit on epoxy resin support.]

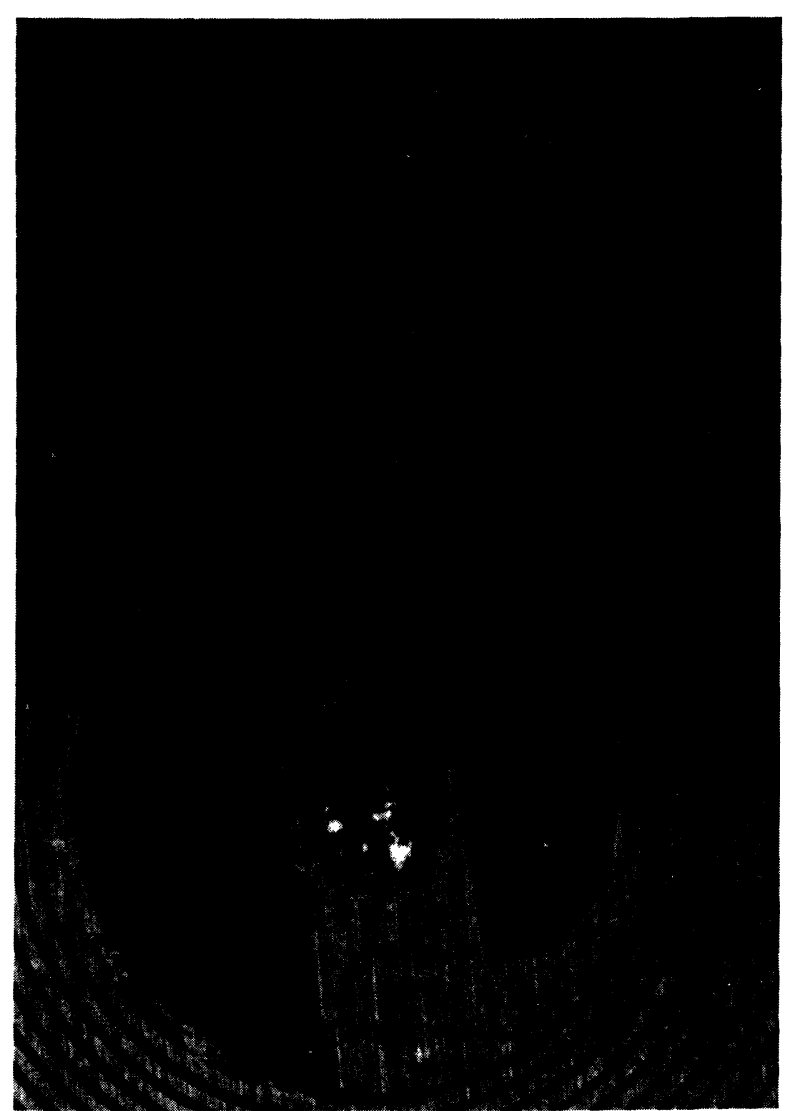

Fig. 2. - Détail des traits et intertraits.

[Detailed part of a flat coil.]

technologie utilisée. Le diamètre des bobines que nous fabriquons est variable. Il est fonction de la taille ainsi que de la nature de l'objet-cible.
2.1.3 Caractérisation électrique. - L'inductance et la résistance de la bobine sont mesurées à l'aide d'un impédancemètre en utilisant un modèle bobine résistance en série négligeant les capacités réparties. Ce modèle est justifié a posteriori par le tracé d'un diagramme de Bode, caractéristique d'un circuit du premier ordre, obtenu à partir d'un filtre passe-bas incluant la bobine plate (Fig. 3).

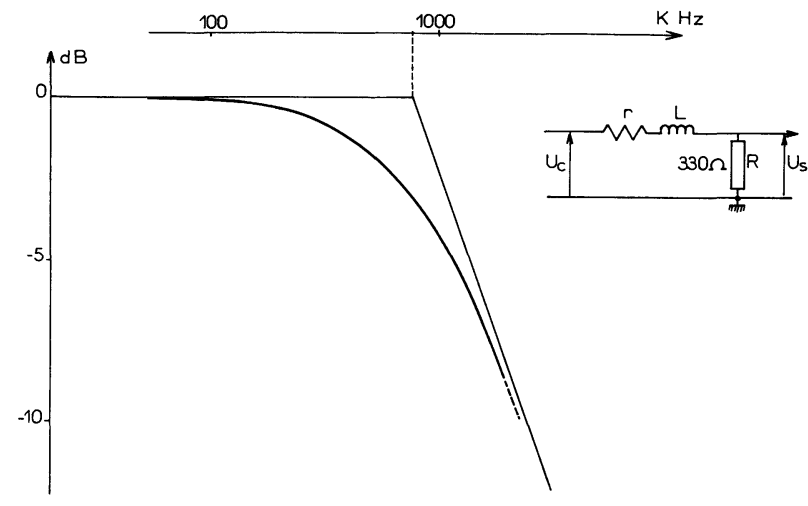

Fig. 3. - Diagramme de Bode.

[Bode diagram.]

Nous présentons dans le tableau I l'évolution respective des valeurs de l'inductance et de la résistance en fonction de divers paramètres physiques et géométriques. Ces résultats concernent un ensemble représentatif d'échantillons réalisés au Laboratoire. La lecture du tableau I appelle quelques remarques.

a) Le facteur de qualité d'une self est fonction de $L \omega / r$. Nous avons donc réalisé quelques échantillons sur circuit imprimé double face. Dans ce cas la résistance est la somme des résistances et l'inductance est donnée par $L=L_{1}+L_{2}+k \sqrt{L_{1} L_{2}}$ où $k$ est un coefficient de couplage. Si celui-ci est satisfaisant, la résistance $r$ croît moins vite que l'inductance $L$.

Le tableau I montre qu'un capteur de $50 \mu \mathrm{m}$ d'épaisseur permet d'obtenir un coefficient de couplage maximum.

b) Le procédé de fabrication des bobines est très fiable. A partir d'un masque ayant une largeur de trait et d'intertrait fixé, on peut tirer un grand nombre de circuits sans que la précision obtenue sur la largeur des traits et intertraits soit fortement altérée. Elle est de l'ordre de $4 \%$.

\subsection{ModÉLISATION EN ABSENCE DE CIBLE.}

2.2.1 Carte du champ. - Une simulation effectuée à partir du logiciel Flux 2D montre qu'on peut parfaitement assimiler la spirale à une distribution uniforme puisque les courbes présentées sur les figures 4 et 5 sont pratiquement superposables. En fait cette approximation n'est valable que si la distance $\mathrm{AA}^{\prime}$ 
Tableau 1. - Caractéristiques physiques et électriques de quelques échantillons.

[Physical and electrical characteristics of some flat coils.]

\begin{tabular}{|l|c|c|c|c|c|c|c|c|c|}
\hline & $N$ & $\begin{array}{c}\text { Epaisseur } \\
\text { support } \\
\text { mm }\end{array}$ & $\begin{array}{c}\varnothing \\
\text { ext. } \\
\text { de la } \\
\text { spirale } \\
\text { int. }\end{array}$ & \multicolumn{2}{|c|}{$\begin{array}{c}\text { Largeur } \\
\text { Trait }\end{array}$} & Inter. T & $L(\mu \mathrm{H})$ & $r(\mathrm{r})$ & $k$ \\
\hline Résine Epoxy & $1 \times 41$ & 1,6 & 20 & 0 & 122 & 78 & 18 & 9 & - \\
\hline Résine Epoxy & $2 \times 41$ & 1,6 & 20 & 0 & 122 & 78 & 55 & 18 & 1,06 \\
\hline Kapton & $2 \times 41$ & 0,125 & 20 & 0 & 122 & 78 & 69 & 18 & 1,83 \\
\hline Kapton & $2 \times 41$ & 0,05 & 20 & 0 & 122 & 78 & 72 & 18 & 2 \\
\hline Résine Epoxy & $1 \times 36$ & 1,6 & 75 & 38 & 334 & 130 & 93,4 & 11,8 & - \\
\hline Résine Epoxy & $1 \times 48$ & 1,6 & 75 & 27 & 329 & 134 & 130 & 12,6 & - \\
\hline Résine Epoxy & $1 \times 61$ & 1,6 & 75 & 16 & 339 & 129 & 154 & 14 & - \\
\hline Résine Epoxy & $1 \times 65$ & 1,6 & 75 & 12 & 340 & 130 & 154 & 16 & - \\
\hline Résine Epoxy & $1 \times 16$ & 1,6 & 100 & 72,5 & 680 & 164 & 37 & 3,6 & - \\
\hline Résine Epoxy & $2 \times 16$ & 1,6 & 100 & 72,5 & 680 & 164 & 110 & 7 & 1,2 \\
\hline
\end{tabular}

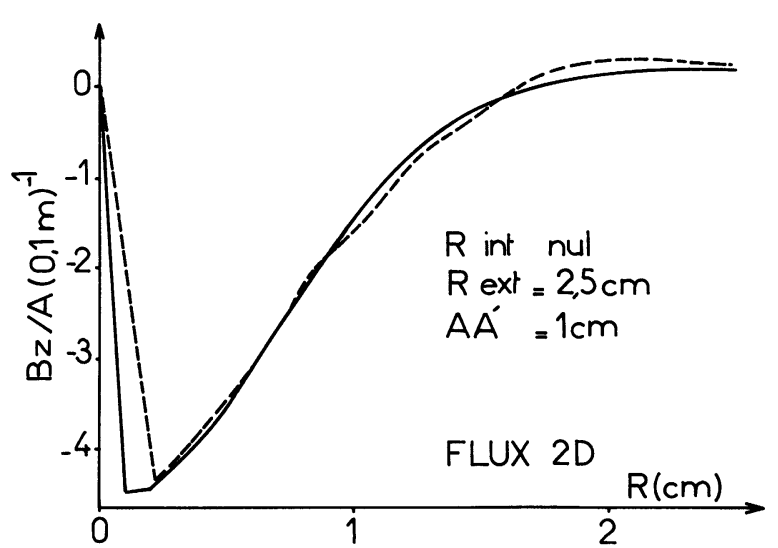

Fig. 4. - Composante normale $B_{z}$ de l'induction le long d'un rayon de la bobine. - Distribution uniforme de courant. ----- Bobine constituée de 44 spires.

[Normal component of induction $B_{\mathrm{z}}$ along one radius of the coil. —Uniform current distribution; --.--. 44 turns coil.]

séparant le plan des spires du point où on mesure le champ est au moins supérieure à 10 fois la largeur de l'intertrait de la bobine plate.

Si l'on tient compte de cette simplification, on peut obtenir à partir de la loi de Biot et Savart les formes littérales de l'induction magnétique créée par la bobine dans un plan parallèle à celle-ci.

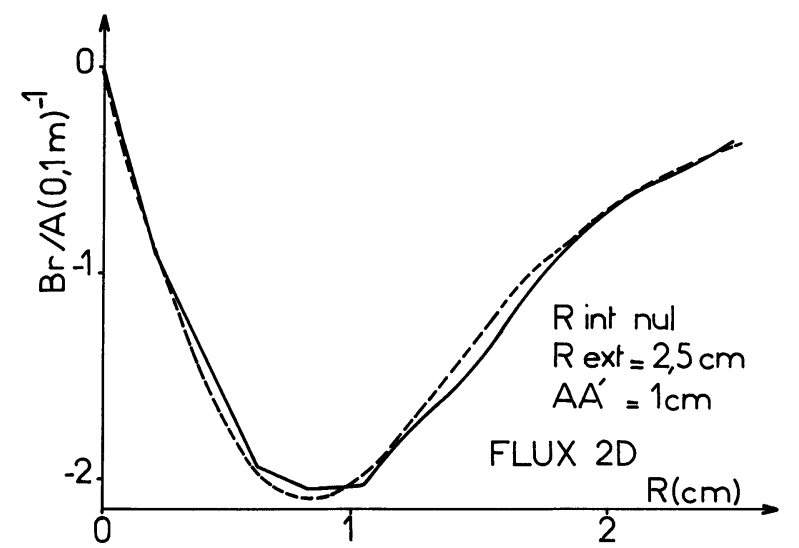

Fig. 5. - Composante radiale $B_{\mathrm{r}}$ de l'induction le long d'un rayon de la bobine. Distribution uniforme de courant. ------ Bobine constituée de 44 spires.

[Radial component of induction $B_{\mathrm{r}}$ along one radius of the coil. —_ Uniform current distribution; --.-- 44 turns coil.]

Soit :

$$
\begin{aligned}
B_{\mathrm{r}}= & -\frac{\mu_{0}}{2 \Pi} \frac{I}{r_{2}-r_{1}} \\
& \int_{0}^{\Pi} \int_{r_{1}}^{r_{2}} \frac{z r \cos \alpha}{\left(h^{2}+r^{2}+z^{2}-2 h r \cos \alpha\right)^{3 / 2}} \mathrm{~d} \alpha \mathrm{d} r(\mathrm{I})
\end{aligned}
$$




$$
\begin{aligned}
B_{\mathrm{z}} & =\frac{\mu_{0}}{2 \Pi} \frac{I}{r_{2}-r_{1}} \\
& \int_{0}^{\Pi} \int_{r_{1}}^{r_{2}} \frac{r^{2}-h r \cos \alpha}{\left(h^{2}+r^{2}+z^{2}-2 h r \cos \alpha\right)^{3 / 2}} \mathrm{~d} \alpha \mathrm{d} r
\end{aligned}
$$

où

$B_{\mathrm{r}}$ est la composante radiale de l'induction

$B_{\mathrm{z}}$ est la composante normale

$r_{1}$ et $r_{2}$ sont respectivement les rayons intérieur et extérieur de la bobine

$z$ est la distance du plan de la bobine au plan de mesure

I est l'intensité totale du courant dans la nappe

$h$ est l'abscisse du point de calcul le long du rayon

$B_{\mathrm{z}}$ et $B_{\mathrm{r}}$ sont des fonctions de $r_{1}, r_{2}, z, h$ et $I$.

Nous avons écrit un logiciel permettant d'effectuer le calcul de I et II. Nos résultats ont été comparés à ceux obtenus à partir de FLUX 2D.

Nous donnons comme exemple (Fig. 6) le résultat du calcul de la composante normale $B_{\mathrm{z}}$ de l'induction magnétique créée par la bobine le long de droites radiales parallèles à son plan situées à 0,$5 ; 1$ et $5 \mathrm{~cm}$. Dans ce calcul les constantes $r_{1}, r_{2}$ et $I$ ont respectivement les valeurs $2 \mathrm{~cm}, 5 \mathrm{~cm}$ et $1 \mathrm{~A}$.

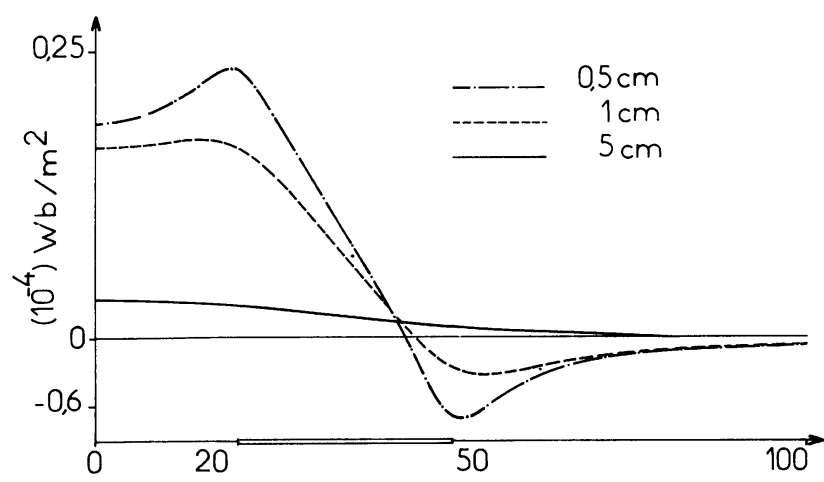

Fig. 6. - Composante $B_{\mathrm{z}}$ d'après nos calculs.

[The $B_{\mathrm{z}}$ component from our calculation.]

Une étude systématique de la répartition de $B_{\mathrm{z}}$ et $B_{\mathrm{r}}$ en fonction de différents paramètres géométriques est en cours. Elle sera publiée ultérieurement.

2.2.2 Calcul du coefficient de self-induction. - Le calcul du coefficient de self-induction de la bobine est indépendant du précédent. On considère cette fois que la bobine est constituée d'un ensemble de $N$ spires circulaires concentriques. Le calcul de l'inductance $L$ consiste à ajouter les coefficients de l'inductance de ces $N$ spires isolées aux $N^{2}$ coefficients d'induction mutuelle de chaque spire sur les spires voisines. On calcule donc :

$$
L_{\mathrm{ii}}=\mu_{0}\left(\frac{r_{\mathrm{i}}}{4}+\left(2 r_{\mathrm{i}}-\rho\right)\left[\left(1-\frac{k^{2}}{2}\right) J_{1}-J_{2}\right]\right)
$$

et

$$
L_{\mathrm{ij}}=\mu_{0}\left(r_{\mathrm{i}}+r_{\mathrm{j}}\right)\left[\left(1-\frac{k^{\prime 2}}{2}\right) J_{1}-J_{2}\right]
$$

où $r_{\mathrm{i}}$ et $r_{\mathrm{j}}$ sont les rayons des spires.

$\rho$ est le rayon du conducteur constituant la spire. Pour des raisons de simplification nous prenons ici une section circulaire plutôt que la section rectangulaire de la bobine réelle; l'incidence sur le résultat du calcul reste faible à condition de considérer des surfaces sensiblement égales. Les valeurs de $k$ et $k^{\prime}$ sont les suivantes :

$$
k=\frac{2 \sqrt{r_{\mathrm{i}}\left(r_{\mathrm{i}}-\rho\right)}}{2 r_{\mathrm{i}}-\rho} \quad k^{\prime}=\frac{2 \sqrt{r_{\mathrm{i}} r_{\mathrm{j}}}}{r_{\mathrm{i}}+r_{\mathrm{j}}}
$$

$J_{1}$ et $J_{2}$ sont les intégrales de Legendre de coefficient $k$ ou $k^{\prime}[6]$.

Dans le tableau II, on peut comparer les valeurs mesurées de l'inductance à celles calculées à partir du programme décrit ci-dessus. Les bobines plates considérées ici ont toutes un diamètre extérieur de $75 \mathrm{~mm}$ et l'épaisseur de cuivre sur le support en résine époxy est de $35 \mu \mathrm{m}$. On peut constater que l'accord entre les valeurs expérimentales et théoriques est très correct. En outre, il ressort de l'ensemble des calculs numériques effectués que l'influence de la largeur du trait sur la valeur de l'inductance est très faible. On ne modifiera cette variable de façon expérimentale qu'à des fins d'optimisation de l'ensemble capteur-conditionneur.

Tableau II. - Valeurs expérimentales et calculées du coefficient de self-induction.

[Experimental and calculated values of the self-induction coefficient.]

\begin{tabular}{|l|c|c|c|c|c|}
\hline$\phi_{\text {int }}(\mathrm{mm})$ & 12 & 16 & 27 & 33 & 38 \\
\hline Nombre spires & 65 & 61 & 48 & 43 & 36 \\
\hline$L$ mesurée & 154 & 154 & 132 & 112 & 93 \\
\hline$L$ calculée & 147,7 & 145 & 121,2 & 111,9 & 90 \\
\hline
\end{tabular}

\section{Montage en capteur.}

\subsection{RÉALISATION ET CARACTÉRISATION.}

3.1.1 Oscillateurs. - Le conditionneur du capteur monté à partir de la bobine qui vient d'être décrite est un oscillateur. Ceux que nous avons utilisés ont été tous réalisés à partir des circuits intégrés commerciaux suivants :

a) TDA 0159 - Thomson-EFCIS (résistance négative). Bien que ce circuit soit plus spécialement conçu pour utiliser sa sortie TTL, il permet de réaliser un 
oscillateur stable en température qui donne satisfaction;

b) TCA 305 - Siemens (oscillateur Meissner).

Le TCA 305 autorise la mise à la masse d'une extrémité de la bobine. En outre une entrée de référence permet d'agir sous certaines conditions sur l'amplitude des oscillations [7].

c) MC 1550 - Motorola (contre-réaction).

Ce circuit a la particularité de donner accès au collecteur d'un des transistors de l'étage différentiel [8]. De plus on peut agir sur l'amplitude des oscillations de par l'entrée AGC (correction automatique de gain).

Ces trois montages différents permettent tous d'obtenir une gamme de fréquences d'oscillations comprise entre 0,8 et $1,5 \mathrm{MHz}$ qui correspond à nos fréquences de travail. Celles-ci résultent évidemment d'un compromis dans lequel interviennent la capacité de l'oscillateur et les caractéristiques de la bobine. Les limitations sont en fait de deux ordres :

- la capacité de l'oscillateur ne doit pas être trop faible si on la compare à l'ordre de grandeur des capacités parasites des bobines de diamètre important,

- la fréquence de travail doit être suffisamment élevée pour que la sensibilité du capteur reste dans un domaine acceptable.

3.1.2 Banc de mesures. - Nous utilisons un banc de mesures "Microcontrôle » pour relever les différentes caractéristiques du capteur. La cible et l'élément sensible (bobine) sont disposés dans deux plans parallèles. Trois vis micrométriques permettent des déplacements selon les axes $x, y, z$ (Fig. 7). Le mouvement de ces vis est assuré par des moteurs pas à pas contrôlés par un système à microprocesseur qui délivre à tout moment sur les deux ports d'un PIA la position relative capteur-cible. La fréquence de l'oscillateur est lue sur un fréquencemètre équipé d'une carte IEEE par un microordinateur Goupil III. Celui-ci gère l'ensemble de l'appareillage d'acquisition, la procédure de poignée de mains avec le système à microprocesseur; il contrôle le bus IEEE auquel est relié le fréquencemètre et un traceur de courbes.

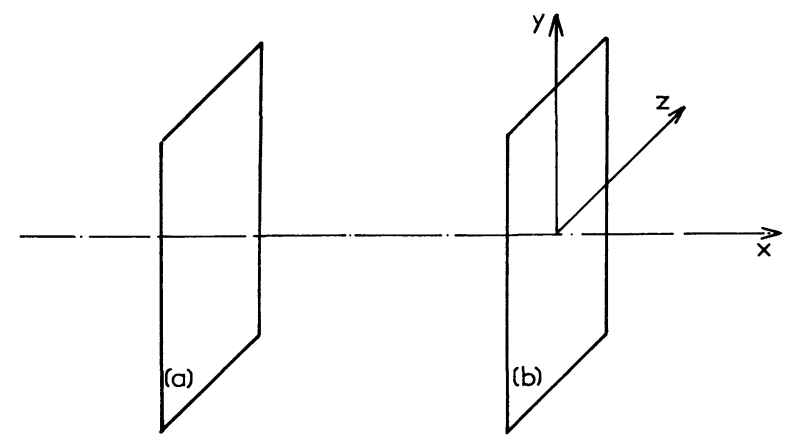

Fig. 7. - Position relative du capteur (a) et de la cible (b).

[Relative location of sensor (a) and target (b)].

REVUE DE PHYSIQUE APPLIQUÉE. - T. 21, № 7, JULLLET 1986
Nous travaillons habituellement avec un déplacement élémentaire des vis micrométriques de $20 \mu \mathrm{m}$. Une acquisition de fréquence est faite après chacun de ces déplacements élémentaires, et le fichier obtenu peut ensuite être traité par microordinateur.

\section{2 RÉSULTATS}

3.2.1 Relation fréquence-proximité. - Les figures 8 et 9 montrent les courbes caractéristiques de la réponse du capteur en fonction de la distance ciblecapteur. La cible est une tôle d'acier de forme carrée $\left(200 \times 200 \mathrm{~mm}^{2}\right)$ et d'épaisseur $1 \mathrm{~mm}$. Les bobines simple face ( 44 spires) et double face $(2 \times 44$ spires $)$ ont un diamètre de $23,5 \mathrm{~mm}$.

Sur ce type de courbe (Fig. 8) ou, pour une plus grande précision, à partir du fichier de données correspondant, on peut définir des paramètres qui permettent une comparaison objective des différents éléments sensibles.

a) La fréquence notée FMAX est définie par extrapolation pour $x=0$.

b) $F_{0}$ est la fréquence «à vide » c'est-à-dire en l'absence de cible. $K_{\mathrm{T}}$ est le rapport FMAX sur $F_{0}$.

c) L'abscisse $x_{\mathrm{L}}$ correspond au point d'intersection de la tangente à la courbe au point (FMAX, 0) avec

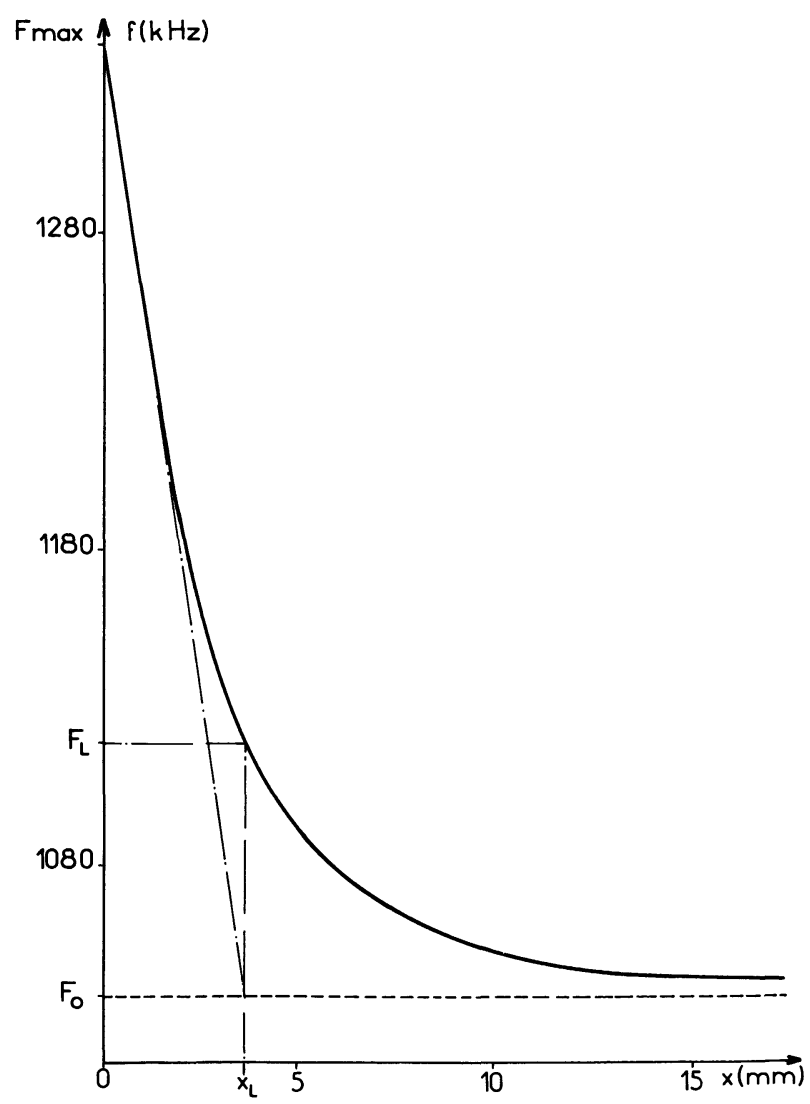

Fig. 8. - Réponse d'un capteur simple face.

[Characteristic of proximity-frequency for a one face coil.] 


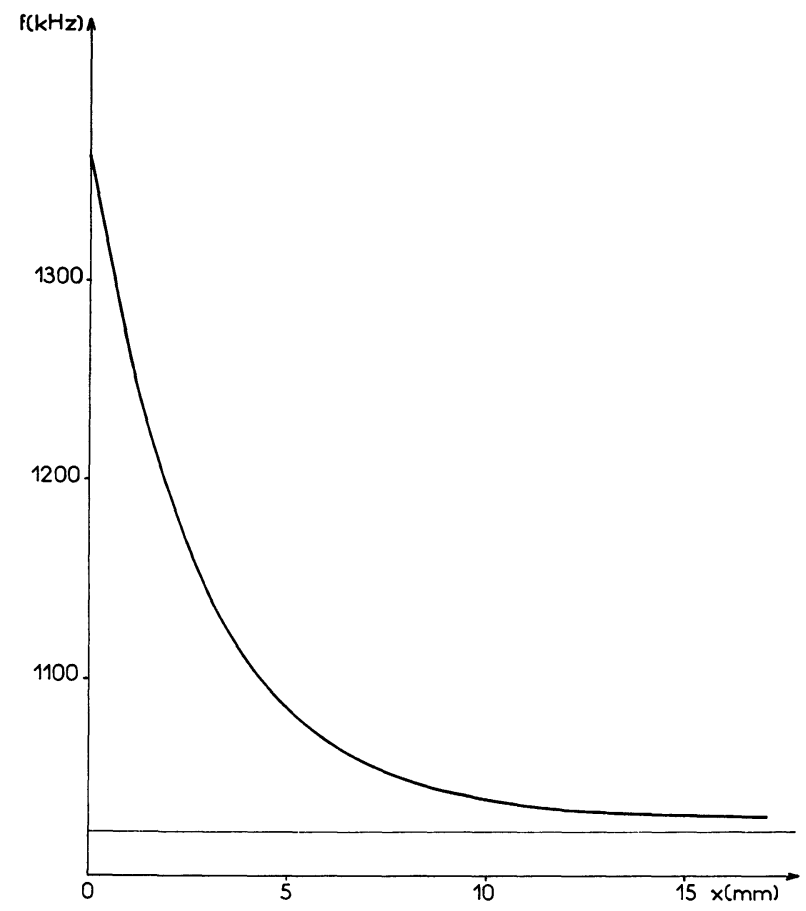

Fig. 9. - Réponse d'un capteur double face.

[Characteristic of proximity-frequency for a two face coil.]

l'horizontale $F_{0}$. La fréquence correspondant à $x_{\mathrm{L}}$ est $F_{\mathrm{L}} . K_{\mathrm{L}}$ est le rapport FMAX sur $F_{\mathrm{L}}$.

d) La sensibilité du capteur définie comme le rapport $\Delta F / \Delta x$ est calculée à partir d'un fichier d'une bobine par une méthode de moindres carrés. Elle peut être calculée en tout point de la courbe.
Le tableau III donne les valeurs des paramètres qui viennent d'être définis pour quelques échantillons de capteurs fabriqués au Laboratoire. Les deux bobines du tableau IV sont réalisées à partir d'un même masque dont on a plus ou moins obturé la partie centrale au tirage. Si on admet que la sensibilité $\Delta F / \Delta x$ est une bonne représentation du pouvoir de détection, on peut considérer que de ce point de vue les deux capteurs ont la même efficacité. Ceci montre que le rôle des spires centrales de la bobine est négligeable et justifie donc la fabrication d'échantillons dont le diamètre intérieur est différent de zéro.

3.2.2 Grandeur d'influence. - L'ensemble bobine conditionneur est sensible à la température.

a) Les condensateurs utilisés dans les différents types d'oscillateurs ont leur diélectrique constitué de verre. L'effet de température peut donc être négligé puisque la variation relative de la capacité $\Delta C / C$ ne dépasse pas $150 \times 10^{-6} /{ }^{\circ} \mathrm{C}$. La dérive en fréquence du capteur est donc principalement due à l'influence de la température sur l'ensemble constitué par la bobine et les autres composants et circuits de l'oscillateur.

b) Une étude globale du comportement du système a été faite en fonction de la température. A titre indicatif, nous donnons ici les valeurs numériques correspondant à une bobine $(N=40, L=109 \mu \mathrm{H}$, $R=11 \Omega, \phi_{\text {ext }}=75 \mathrm{~mm}, \phi_{\text {int }}=34,5 \mathrm{~mm}$ ) en absence de tôle. La dérive en fréquence due à l'ensemble du système est inférieure à $0,3 \mathrm{kHz} /{ }^{\circ} \mathrm{C}$ pour une réponse de $930 \mathrm{kHz}$, ceci autour de la température ambiante. Un capteur en présence de tôle d'acier (épaisseur

Tableau III. - Valeurs numériques des paramètres caractéristiques d'un échantillon.

[Numerical values of main-coil parameters.]

\begin{tabular}{|lll|c|c|c|c|}
\hline \multicolumn{3}{|c|}{ Echantillons } & $K_{\mathrm{T}}$ & $K_{\mathrm{L}}$ & $x_{\mathrm{L}} \mathrm{mm}$ & $\frac{F_{\max }-F_{\mathrm{L}}}{x_{\mathrm{L}}} \mathrm{kHz} / \mathrm{mm}$ \\
\hline$N=44$ & $\varnothing_{\text {ext }}=23,5 \mathrm{~mm}$ & $\varnothing_{\text {int }}=0 \mathrm{~mm}$ & 1,28 & 1,20 & 3,9 & 57 \\
\hline$N=2 \times 44$ & $\varnothing_{\text {ext }}=23,5 \mathrm{~mm}$ & $\varnothing_{\text {int }}=0 \mathrm{~mm}$ & 1,33 & 1,22 & 3,5 & 67,7 \\
\hline$N=65$ & $\varnothing_{\text {ext }}=75 \mathrm{~mm}$ & $\varnothing_{\text {int }}=12 \mathrm{~mm}$ & 2,16 & 1,58 & 4,8 & 188 \\
\hline
\end{tabular}

Tableau IV. - Influence du nombre de spires sur la détection.

[Influence of the number of turns on the detection.]

\begin{tabular}{|l|l|l|l|l|l|l|l|l|l|l|l|l|}
\hline Distance capteur cible en mm & & 10 & 15 & 20 & 25 & 30 & 35 & 40 & 45 & 50 & 55 \\
\hline$N=65 \quad \varnothing_{\text {ext }}=75 \quad \varnothing_{\text {int }}=12(\mathrm{~mm})$ & $\begin{array}{c}\Delta F / \Delta x \\
\text { en } \mathrm{kHz} / \mathrm{mm}\end{array}$ & 27,3 & 11,8 & 6 & 3,3 & 2 & 1,2 & 0,8 & 0,54 & 0,36 & 0,27 \\
\cline { 1 - 8 }$N=40$ & $\varnothing_{\text {ext }}=75$ & $\varnothing_{\text {int }}=35(\mathrm{~mm})$ & & 11 & 5,7 & 3,2 & 1,9 & 1,2 & 0,8 & 0,54 & 0,38 & 0,27 \\
\hline
\end{tabular}


$1 \mathrm{~mm}$, dimensions grandes devant le diamètre de l'élément sensible) et soumis à une variation de température peut présenter une erreur $\Delta x$ lors d'un positionnement statique. Cette erreur fonction de la distance cible-bobine croît lorsqu'on s'éloigne de la tôle. Nous donnons ici pour quelques valeurs de $x$ (distance cible-bobine) les limites supérieures correspondant à $\Delta x$.

$$
\begin{aligned}
& x=1 \mathrm{~cm}, \quad \Delta x<0,01 \mathrm{~mm} /{ }^{\circ} \mathrm{C} \\
& x=3 \mathrm{~cm}, \quad \Delta x<0,2 \mathrm{~mm} /{ }^{\circ} \mathrm{C} \\
& x=5 \mathrm{~cm}, \quad \Delta x<0,7 \mathrm{~mm} /{ }^{\circ} \mathrm{C} \text {. }
\end{aligned}
$$

c) Les utilisations que nous développons au laboratoire pour ce capteur font appel à des mesures dynamiques. Pour un positionnement absolu, avec une grande précision on doit évidemment envisager un tarage.

3.2.3 Détection de bord de tôle et de trous. - a) Bord de tôle. La bobine se déplace suivant l'axe $z$ dans un plan parallèle à la tôle. La distance $x$ entre les deux plans est constante.

La figure 10 montre une courbe type $F=f(z)$, obtenue avec nos capteurs pour la détection de bord de tôle avec $x_{0}=2,4 \mathrm{~mm}$. Il s'agit ici d'un échantillon double face déjà cité $(2 \times 44$ spires $\varnothing 23,5 \mathrm{~mm})$. La variation maximum de fréquence (en présence de tôle et hors tôle) est de l'ordre de $140 \mathrm{kHz}$. La partie linéaire de la courbe correspond à une sensibilité $\Delta F / \Delta z$ de l'ordre de $15,6 \mathrm{kHz} / \mathrm{mm}$.

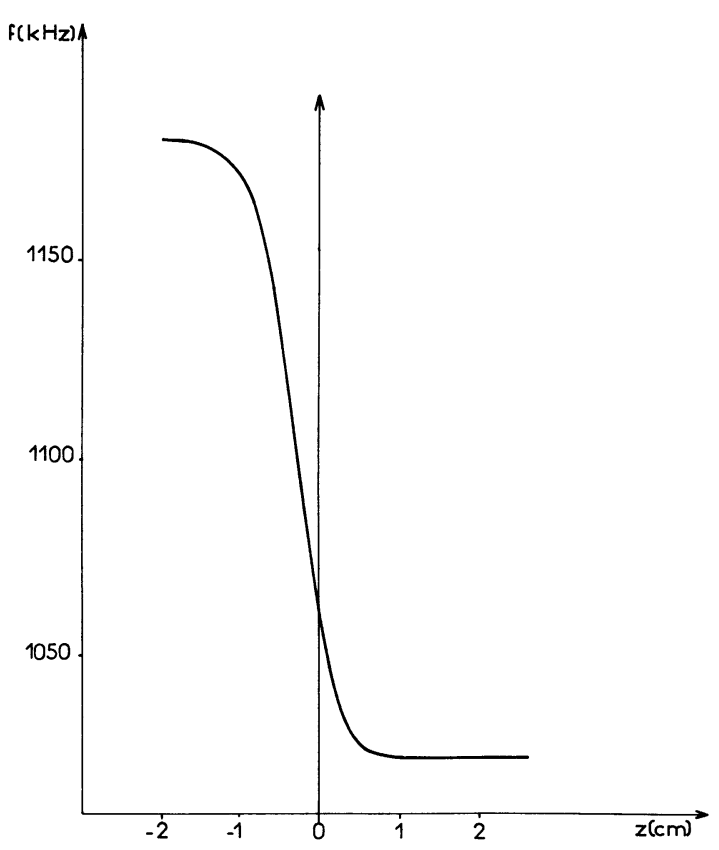

Fig. 10. - Détection d'un bord de tôle d'acier.

[Detection of an edge of iron sheet.] b) Détection d'un trou circulaire. Nous utilisons le même montage que ci-dessus. Les centres du trou et de la bobine sont alignés sur un même axe qui sera pris comme origine. La figure 11 montre les courbes caractéristiques (puits de fréquence) obtenues à partir du même capteur. La profondeur et la forme de la courbe de réponse dépendent du rapport relatif des diamètres de la bobine et du trou, ainsi que de la distance capteur-tôle. En particulier, on peut obtenir des courbes présentant une absence de pic médian $(\varnothing$ trou plus grand que $\varnothing$ capteur).

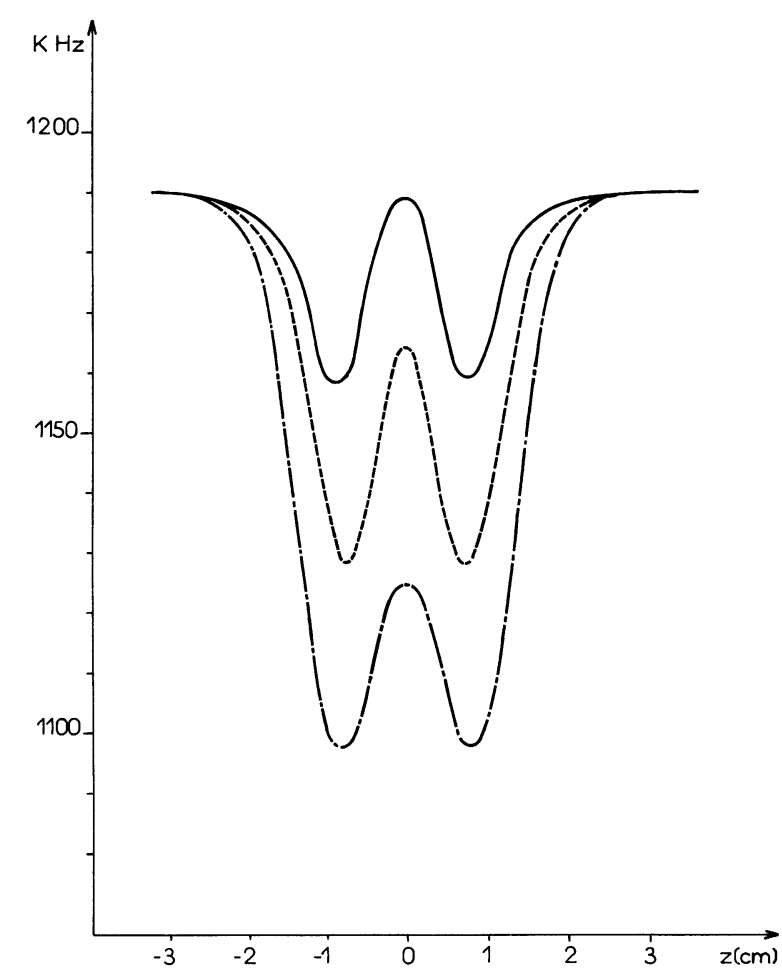

Fig. 11. - Présence d'un trou dans une tôle d'acier. -.-.-.-. diamètre du trou $20,1 \mathrm{~mm}$; -------- diamètre du trou $15,2 \mathrm{~mm}$; - diamètre du trou $10,0 \mathrm{~mm}$.

[Frequency variation observed according to the location of the sensor with regard to the hole. -.-.-.-. The hole diameter is $20.1 \mathrm{~mm}$; ------ The hole diameter is $15.2 \mathrm{~mm}$; The hole diameter is $10.0 \mathrm{~mm}$ ].

Une asymétrie plus ou moins marquée peut aussi refléter le parallélisme imparfait entre les plans de la tôle et la bobine.

Ces caractéristiques nous ont poussé à développer des algorithmes de détection et de position automatique au centre d'un trou ou sur le bord d'une tôle [9].

Les travaux détaillés concernant la précision de ces positionnements seront publiés ultérieurement. On peut cependant indiquer rapidement que l'on sait reconnaître la position de l'axe d'un trou de quelques centimètres de diamètre, avec une imprécision $\Delta x$ 
toujours inférieure à $10 \mu \mathrm{m}$, (distance tôle-capteur $=$ $3 \mathrm{~mm}$ ). Ceci sans prendre de précaution particulière quant à la température. La méthode de mesure est ici une méthode dynamique.

Les logiciels correspondants ont été implantés sur carte microprocesseur.

\section{Conclusion.}

L'étude précédente montre qu'il est possible d'utiliser de telles bobines plates intégrées à un oscillateur pour la détection et la localisation d'une cible métallique. Ce procédé outre qu'il est bon marché est très facile à réaliser industriellement.

Le faible poids de la bobine autorise l'incorporation de l'électronique de traitement et de commande au sein du capteur, L'ensemble pourrait par exemple être placé à l'extrémité d'un bras de robot.

Nous travaillons à développer et optimiser de tels capteurs pour diverses applications industrielles.

\section{Bibliographie}

[1] Asch, G., Les capteurs en instrumentation industrielle (Dunod, Paris) 1982.

[2] Garrat, J. D., J. Phys. E. : Sci. Instrum. 12 (1979) 563.

[3] Placko, D., Dispositif d'analyse de profil utilisant des capteurs à courant de Foucault. Thèse de $3 \mathrm{e}$ cycle. Orsay, 1984.

[4] Kaplan, B. Z., Mishal, R., Fetman, A. and Gressel, C., Rev. Sci. Instrum. 49 (11) (1978) 1583.

[5] Clergeot, H., Monteil, F., Placko, C., Detriche, J. M., Dispositif d'analyse de profil utilisant des capteurs à courant de Foucault. AFCET Besançon, 1983.
[6] Durand, E., Electrostatique et Magnétostatique (Masson, Paris) 1953.

[7] Strehle, G., Zulauf, J. M. Exemple d'application No 9. Référence B 9.102, 1978. Siemens S. A. Division composants B.P. 109, 93203 Saint-Denis.

[8] Floutier, D., Electron. Appl. 50 (11) (1979) 79.

[9] Villermain-LeColier, G., Billat, A., SENSOR'85 Transducer-Technik. Karlsruhe. Conference Proceedings (4) 1985, 621. 